\title{
A Combinatorial Interpretation for the Schett Recurrence on the Jacobian Elliptic Functions
}

\section{By Dominique Dumont}

\begin{abstract}
The coefficients introduced by Alois Schett containing the Taylor series expansions of the Jacobian elliptic functions are proved to count certain classes of permutations.
\end{abstract}

1. Introduction. Let $D$ be the derivative operator on the polynomials in three variables defined by

$$
D x=y z, \quad D y=z x, \quad D z=x y .
$$

The polynomials $S_{m}(x, y, z)(m \geqslant 0)$ introduced by Schett [3] in a slightly different form (called Schett polynomials in this paper) can be defined by induction as follows:

$$
\left\{\begin{array}{l}
S_{0}(x, y, z)=x, \\
S_{m}(x, y, z)=D S_{m-1}(x, y, z) \quad(m \geqslant 1) .
\end{array}\right.
$$

The first values of $S_{m}$ are

$$
\begin{aligned}
& S_{1}(x, y, z)=y z, \\
& S_{2}(x, y, z)=y D z+z D y=x y^{2}+x z^{2}, \\
& S_{3}(x, y, z)=y^{3} z+y z^{3}+4 x^{2} y z, \\
& S_{4}(x, y, z)=x y^{4}+14 x y^{2} z^{2}+x z^{4}+4 x^{3} y^{2}+4 x^{3} z^{2} .
\end{aligned}
$$

Each $S_{m}$ has total degree $(m+1)$ and its coefficients are positive integers. When $m$ is even (respectively, odd), $S_{m}$ is odd (respectively, even) in $x$ and even (respectively, odd) in $y$ and $z$. Accordingly, for

$$
\left\{\begin{array}{l}
\text { for } m=2 n, \quad S_{2 n}(x, y, z)=\sum_{i, j \geqslant 0} a_{2 n, i, j} x^{2 i+1} y^{2 j} z^{2 n-2 i-2 j}, \\
\text { for } m=2 n+1, \quad S_{2 n+1}(x, y, z)=\sum_{i, j \geqslant 0} a_{2 n+1, i, j} x^{2 i} y^{2 j+1} z^{2 n-2 i-2 j+1} .
\end{array}\right.
$$

With $n$ denoting the integral part of $m(n=[m / 2])$ relation (1) yields 
(2)

$$
\left\{\begin{aligned}
& \text { for } m \geqslant 1, i+j<0 \text { or } i+j>n, \quad a_{m, i, j}=0, \quad a_{1,0,0}=1, \\
& a_{2 n, i, j}=(2 j+1) a_{2 n-1, i, j}+(2 i+2) a_{2 n-1, i+1, j-1} \\
&+(2 n-2 i-2 j+1) a_{2 n-1, i, j-1}, \\
& a_{2 n+1, i, j}=(2 i+1) a_{2 n, i, j}+(2 j+2) a_{2 n, i-1, j+1}+(2 n-2 i-2 j+2) a_{2 n, i-1, j}
\end{aligned}\right.
$$

Now using (2) it is straightforward to obtain the first values of the Schett polynomial coefficients. (For $S_{7}$ and $S_{8}$, see [5].)

\section{TABLE 1}

\begin{tabular}{|c|c|c|c|c|c|c|c|c|c|c|c|c|c|c|c|c|c|}
\hline$l$ & 0 & 1 & $j i$ & 0 & 1 & $i$ & 0 & 1 & 2 & 1 & $\begin{array}{ll}0 & 1 \\
\end{array}$ & 2 & $j i$ & 0 & 1 & 2 & 3 \\
\hline 0 & 1 & 0 & 0 & 1 & 4 & 0 & 1 & 4 & 0 & 0 & 144 & 16 & 0 & 1 & 44 & 16 & 0 \\
\hline \multirow[t]{3}{*}{1} & 1 & 0 & 1 & 1 & 0 & 1 & 14 & 4 & 0 & 1 & 1444 & 0 & 1 & 135 & 328 & 16 & 0 \\
\hline & & & & & & 21 & 1 & 0 & 0 & 2 & 10 & 0 & 2 & 135 & 44 & 0 & 0 \\
\hline & & & & & & & & & & & & & 3 & 1 & 0 & 0 & 0 \\
\hline & $, i, j$ & & & & & & $, i, j$ & & & & $, i, j$ & & & & & & \\
\hline
\end{tabular}

On the other hand, the Taylor series expansions of the Jacobian elliptic functions sn, cn and dn, read

$$
\begin{aligned}
\operatorname{sn}(u, k)= & u-\left(1+k^{2}\right) \frac{u^{3}}{3 !}+\left(1+14 k^{2}+k^{4}\right) \frac{u^{5}}{5 !} \\
& -\left(1+135 k^{2}+135 k^{4}+k^{6}\right) \frac{u^{7}}{7 !}+\cdots \\
\operatorname{cn}(u, k)= & 1-\frac{u^{2}}{2 !}+\left(1+4 k^{2}\right) \frac{u^{4}}{4 !}-\left(1+44 k^{2}+16 k^{4}\right) \frac{u^{6}}{6 !}+\cdots \\
\operatorname{dn}(u, k)= & 1-k^{2} \frac{u^{2}}{2 !}+\left(4+k^{2}\right) \frac{u^{4}}{4 !}-\left(16+44 k^{2}+k^{4}\right) \frac{u^{6}}{6 !}+\cdots
\end{aligned}
$$

The main result by Schett was to prove that the coefficients of these expansions are precisely equal to the coefficients $a_{m, i, 0}$ and $a_{m, 0, j}$ that occur in the first rows and columns of the previous tables. Schett also noticed that

$$
\sum_{i, j \geqslant 0} a_{m, i, j}=m !
$$

holds for every $m \geqslant 1$. This strongly suggests that the set of integers $\left(a_{m, i, j}\right)_{(i, j \geqslant 0)}$ be the distribution of a bivariate statistic $(X, Y)$ defined on the permutation group $\widetilde{G}_{m}$. The purpose of this paper is precisely to construct such a statistic.

Let $\sigma$ be an element of $\mathfrak{G}_{m}$, and denote by $\sigma^{-1}$ its inverse in $\mathfrak{S}_{m}$. Then a cycle peak of $\sigma$ is defined to be an integer $k$ with the following properties:

$$
2 \leqslant k \leqslant m, \quad \sigma(k) \neq k, \quad \sigma(k)<k \quad \text { and } \quad \sigma^{-1}(k)<k .
$$

For example, with $\sigma=\left(\begin{array}{llllll}1 & 2 & 3 & 4 & 5 & 6 \\ 3 & 2 & 4 & 1 & 6 & 5\end{array}\right)$ the cycles are (1 34 ), (2), and (5 6 ) and the only cycle peaks 4 and 6 . 
Let $X(\sigma)$ (respectively, $Y(\sigma)$ ) be the number of odd (respectively, even) cycle peaks of $\sigma$. Then our main theorem reads:

THEOREM. Each coefficient $a_{m, i, j}$ is equal to the number of permutations in $\Xi_{m}$ having $i$ odd cycle peaks and $j$ even cycle peaks, i.e.,

$$
a_{m, i, j}=\left|\left\{\sigma \in \widetilde{\Xi}_{m}: X(\sigma)=i, Y(\sigma)=j\right\}\right| .
$$

Example. For $m=3$ the permutation (1) (2) (3) has no cycle peak; (1 2 ) (3) has only one even cycle peak; each of the four remaining ones (1 $\underline{3})$ (2), (1) (2 $\underline{3})$, (1 $22 \underline{3}),\left(\begin{array}{lll}1 & 3 & 2\end{array}\right)$ has only one odd cycle peak. (See Table 1 for $m=3$.)

The above theorem has several consequences. With $i=0$ (respectively, $j=0$ ) relation (3) provides new combinatorial interpretations for the coefficients $\operatorname{sn}(u, k)$ (respectively, $\operatorname{cn}(u, k)$ and $\operatorname{dn}(u, k)$ ) (see Corollary 1$)$. On the other hand, as the Jacobi elliptic functions are simply related to the Euler numbers $E_{m}(m \geqslant 1)$, our approach will also lead to a new combinatorial interpretation for the coefficients $E_{m}$ (see Corollaries 2 and 4).

2. Proof of the Theorem. If $\sigma$ is in $\Xi_{m}$, consider the permutation $\tau$ obtained from $\sigma$ by removing $m$ from its cycle. In other words, if $\sigma(m)=m$, then $\tau$ is the restriction of $\sigma$ to $\{1,2, \ldots, m-1\}$; if $\sigma(m)<m$, let $\tau\left(\sigma^{-1}(m)\right)=\sigma(m)$ and for $i \neq \sigma^{-1}(m), \tau(i)=\sigma(i)$.

Conversely, if $\tau$ is in $\widetilde{G}_{m-1}$, then $\tau$ will be extended to a permutation $\sigma$ in $\widetilde{\Xi}_{m}$ in $m$ different ways. Choose an integer $k(1 \leqslant k \leqslant m)$. If $k=m$, let $\sigma(m)=m$. If not, let $\sigma(k)=m$ and $\sigma(m)=\tau(k)$. We say that $m$ has been inserted into the cycle of $k$ on the right of $k$ (on the left of $k$ would be: $\sigma\left(\tau^{-1}(k)\right)=m$ and $\sigma(m)=k$ ). For the other elements let $\sigma(i)=\tau(i)$.

The only problem is to see how the numbers of odd and even cycle peaks are changed when we go from $\tau$ to $\sigma$. Assume $m=2 n$.

(a) First examine the case when the insertion of $2 n$ leaves the numbers of odd and even cycle peaks invariant. Start with $\tau$ having $i$ odd and $j$ even cycle peaks. For $j$ not to be changed, the element $2 n$ has to be inserted on the right or on the left of an even peak of $\tau$, or it must become a fixed point of $\sigma$, i.e., $\sigma(2 n)=2 n$. There are exactly $(2 j+1)$ possibilities to do so.

(b) If we want to remove one odd cycle peak, we must put $2 n$ on either side of some odd peak of $\tau$. If $\tau$ has $(i+1)$ odd and $j-1$ even peaks, there exist $(2 i+2)$ possibilities to do so and $\sigma$ will have $i$ odd and $j$ even peaks.

(c) The remaining case is to start with $\tau$ having $i$ odd and $j-1$ even cycle peaks and create a new even peak. We have to exclude the $(2(j-1)+1)$ insertions of type (a), and the $2 i$ ones of type (b). There so remain $(2 n-2 i-2 j+1)$ possibilities to add an even cycle peak.

This shows that the number of permutations in $\Im_{m}$ with $i$ odd and $j$ even cycle peaks satisfies recurrence relation (2) in the even case. The proof is quite analogous in the odd case and will be omitted. 
3. Applications. We can now interpret Schett's result on the coefficients $a_{m, i, 0}$ and $a_{m, 0, j}$

COROllaRY 1. (i) The coefficient of $(-1)^{n} k^{2 j} u^{2 n+1} /(2 n+1)$ ! in the Taylor expansion of $\operatorname{sn}(u, k)$ is equal to the number of permutations in $\mathfrak{G}_{2 n}$ (or in $\left.\mathfrak{G}_{2 n+1}\right)$ having $j$ cycle peaks, all even.

(ii) The coefficient of $(-1)^{n} k^{2 i} u^{2 n} /(2 n)$ ! (respectively, $(-1)^{n} k^{2 n-2 i} u^{2 n} /(2 n)$ !) in the Taylor expansion of $\mathrm{cn}(u, k)$ (respectively, $\operatorname{dn}(u, k)$ ) is equal to the number of permutations in $\mathfrak{G}_{2 n-1}$ (or in $\mathfrak{S}_{2 n}$ ) having $i$ cycle peaks all odd. It is also equal to the number of permutations in $\mathfrak{S}_{2 n}$ having $n$ cycle peaks, $i$ among them being odd.

Recall that the Euler numbers $\left(E_{m}\right), m \geqslant 0$, are defined by

$$
\sec x+\tan x=\sum_{m \geqslant 0} E_{m} \frac{x^{m}}{m !} .
$$

It is well known that $\operatorname{cn}(u, 1)=\sec (i u)$ and $\operatorname{sn}(u, 1)=-i \tan (i u)$. Therefore,

COROLLARY 2. The Euler number $E_{m}(m \geqslant 1)$ is equal to the number of permutations in $\mathfrak{G}_{m-1}$ (or in $\mathfrak{G}_{m}$ ) having no cycle peaks of the parity of $m$.

COROLlARY 3 (SchetT). The number of permutations in $\Xi_{m}$ having $k$ run-ups $(0 \leqslant k \leqslant m)$ is equal to the sum $\Sigma_{i+j=k} a_{m, i, j}$

A direct combinatorial proof can be made by noting that $\Sigma_{i+j=k} a_{m, i, j}$ counts the permutations in $\mathfrak{S}_{m}$ having $k$ cycle peaks and using the fundamental transformation of $\mathcal{G}_{m}([2$, p. 13]).

The following interpretation is a refinement of Andre's result on secant numbers [1]. Recall that a permutation $\sigma$ in $\Xi_{2 n}$ is alternating if $\sigma(1)<\sigma(2)>\sigma(3)<\sigma(4)$ $>\cdots>\sigma(2 n-1)<\sigma(2 n)$.

COROllary 4. The coefficient of $(-1)^{n} k^{2 i} u^{2 n} /(2 n)$ ! in the Taylor expansion of $\mathrm{cn}(u, k)$ is equal to the number of alternating permutations in $\mathfrak{G}_{2 n}$ with the property that $\sigma(2 j)$ is odd for exactly $i$ abscissas $2 j$.

This can be seen from the fact that if $i+j=n$, the run-ups are simply rises and so the permutation alternating.

Remarks. (i) The sum $\Sigma_{j} a_{m, i, j}$ was studied by Schett. He showed that it is equal to the number of permutations in $\Xi_{m}$ having $m-i$ peaks (not cycle peaks!). No direct bijection with the permutations having $i$ odd cycle peaks is known.

(ii) The first combinatorial interpretation for the Jacobian elliptic function coefficients was given by Viennot [6] with an entirely different set-up.

The author thanks Professor Schützenberger for having drawn his attention to Schett's papers.

Note Added in Proof. The result stated in Corollary 4 has been obtained independently by P. Flajolet ("Combinatorial aspects of continued fractions," IRIA, 78150Rocquencourt, France). 
Département de Mathématique

Université de Strasbourg

Strasbourg, France

1. D. ANDRE, "Développement de $\sec x$ et $\tan x$," C. R. Acad. Sci. Paris, v. 88, 1879, pp. 965-967.

2. D. FOATA \& M. P. SCHÜT ZENBERGER, Théorie Géométrique des Polynômes Eulériens, Lecture Notes in Math., Vol. 138, Springer-Verlag, Berlin and New York, 1970.

3. A. SCHETT, "Properties of the Taylor series expansion coefficients of the Jacobian elliptic functions," Math. Comp., v. 30, 1976, pp. 143-147.

4. A. SCHETT, Addendum to "Properties of the Taylor series expansion coefficients of the Jacobian elliptic functions," Math. Comp., v. 31, 1977, Microfiche supplement.

5. A. SCHETT, "Recurrence formula of the Taylor series expansion coefficients of the Jacobian elliptic functions," Math. Comp., v. 31, 1977, pp. 1003-1005.

6. G. VIENNOT, "Une interprétation combinatoire des coefficients des développements en série entière des fonctions elliptiques de Jacobi," J. Combinatorial Theory. (To appear.) 